\title{
Clinical Analysis of the Myosure Hysteroscopic Tissue Removal System in 740 Cases of Benign Intrauterine Lesions
}

\section{Jiahui Yong}

the Department of Gynecology and Obstetrics, Central South University, Third Xiangya Hospital

Xiaohui Guo

the Department of Gynecology and Obstetrics, Central South University, Third Xiangya Hospital

Chu Liu

the Department of Gynecology and Obstetrics, Central South University, Third Xiangya Hospital

Jing Yuan

the Department of Gynecology and Obstetrics, Central South University, Third Xiangya Hospital

\section{Yajun Wan}

the Department of Gynecology and Obstetrics, Central South University, Third Xiangya Hospital

\section{Fei Zeng}

the Department of Gynecology and Obstetrics, Central South University, Third Xiangya Hospital

\section{Xin Sun}

the Department of Gynecology and Obstetrics, Central South University, Third Xiangya Hospital

\section{Yimin Yang}

the Department of Gynecology and Obstetrics, Central South University, Third Xiangya Hospital

\section{Shuijing Yi}

the Department of Gynecology and Obstetrics, Central South University, Third Xiangya Hospital

\section{Xiangyang Zeng}

the Department of Gynecology and Obstetrics, Central South University, Third Xiangya Hospital

Songshu Xiao ( $\nabla$ xiaosongshu@csu.edu.cn )

the Department of Gynecology and Obstetrics, Central South University, Third Xiangya Hospital

\section{Research Article}

Keywords: Hysteroscopic tissue removal system, MyoSure, Endometrial Polyps, Submucosal myomas, Retained products of conception

Posted Date: March 2nd, 2021

DOI: https://doi.org/10.21203/rs.3.rs-241108/v1 
License: (c) (i) This work is licensed under a Creative Commons Attribution 4.0 International License. Read Full License 


\section{Abstract}

Objective: To investigate the clinical efficacy of the MyoSure hysteroscopic tissue removal system in the treatment of benign intrauterine lesions.

Methods: Retrospective analysis was performed on the clinical data of 740 patients treated with the MyoSure hysteroscopic tissue removal system for benign intrauterine lesions from September 2014 to December 2018, including 131 cases of submucosal myomas, 85 cases of endometrial polyps and 524 cases of RPOC. The clinical efficacy of MyoSure surgery was analyzed according to patients' intraoperative conditions (intraoperative blood loss, operative time, lesion complete resection rate, and intraoperative complications) and postoperative symptom relief rate.

Results: All 740 patients successfully completed the operation. The intraoperative blood loss was $17.09 \pm 14.87 \mathrm{ml}, 11.78 \pm 10.89 \mathrm{ml}$ and $29.50 \pm 13.74 \mathrm{ml}$ for submucosal myomas, endometrial polyps and RPOC, respectively, and the operative time was $42.37 \pm 19.33 \mathrm{~min}, 20.96 \pm 8.99 \mathrm{~min}$ and $30.47 \pm 14.04 \mathrm{~min}$, respectively. The complete resection rates (CRRs) of type 0 , type $\varangle$ and type $\mathbb{Q}$ lesions and mixed type fibroids were $100.0 \%$ (45/45), 96.00\% (48/50), 85.67\% (13/15) and $75.00 \%(9 / 12)$, respectively. For endometrial polyps and RPOC, the CRRs were $100 \%$ (85/85) and $98.28 \%(515 / 524)$, respectively. No intraoperative complications, such as uterine perforation, were reported with the exception of one patient with RPOC who exhibited TURP syndrome during the operation. The postoperative satisfaction rates for submucosal myomas, endometrial polyps and RPOC were $94.66 \%$ (124/131), 100.0\% (85/85) and $98.85 \%$ (518/524), respectively. The conception rate was $47.91 \%(172 / 359)$, and the live birth rate was $59.30 \%(102 / 172)$.

Conclusions: The MyoSure hysteroscopic tissue removal system is a safe and effective new treatment for benign intrauterine lesions for women of childbearing age.

\section{Introduction}

Submucosal myomas, endometrial polyps, and retained products of conception (RPOC) are common benign intrauterine lesions in women of childbearing age [1]. In addition to causing bleeding symptoms such as menorrhagia, menostaxis or intermenstrual spotting, which may lead to anemia, they may also be associated with secondary infertility or abnormal pregnancy event [2].

Hysteroscopic loop-electrode resectoscopy is commonly used to deal with intrauterine lesions. Electrical resection can effectively remove the lesion, but cervical trauma, uterine perforation and TURP syndrome are severe complications which cannot be ignored. Also, it's difficult to avoid secondary endometrial damage due to the use of electric current and thermal energy during electrical resection [3].

Hysteroscopic morcellation is a recently emerged tissue removal system. The procedure is performed using rotating tube inside the cutting window, and the resected tissue is then aspirated immediately through the device into a collecting pouch, which make the procedure more safe and efficient [4]. The 
TRUCLEAR hysteroscopic morcellator and MyoSure Tissue Removal System are respectively the first and second generation hysteroscopic morcellator. Compared to TRUCLEAR, MyoSure is equipped with a hysteroscope with a smaller outer diameter of $6.25 \mathrm{~mm}$, and a faster rotated inner blade at speeds as high as $6000 \mathrm{rpm}$. Also, MyoSure can be compatible with all currently available fluid management systems [5]. Previous clinical studies on MyoSure have proved the high efficacy and safety of the procedure $[9-12,15]$.

Currently, the effect of hysteroscopic morcellation on outcomes of gynecology and obstetrics in patients with intrauterine lesions is not clear. Therefore, the purpose of this study is not only to evaluate the clinical efficacy of MyoSure through a relatively large amount of cases, but also to investigate the effect of MyoSure on outcomes of gynecology and obstetrics in patients of childbearing age.

\section{Materials And Methods}

\section{Study site}

The research was conducted at the Changsha institute of Gynecology and Obstetrics, The Third Xiangya Hospital, Central South University, Changsha, Hunan, China. The study was approved by the Ethics Committee of Third Xiangya Hospital. We confirm that all experiments were performed in accordance with relevant named guidelines and regulations. We confirm that informed consent was obtained from all participants and their legal guardians.

\section{Patient selection}

This is a retrospective cohort study enrolling women who underwent the MyoSure procedure for intrauterine lesions at the Third Xiangya Hospital of Central South University, spanning the period September 1, 2014 to December 31, 2018. The study received approval from the Research Ethics Committee of the institution. The inclusion criteria consisted of the following: (1) identification of intrauterine lesions (endometrial polyps, submucosal myomas and RPOC) by preoperative ultrasonographic findings and/or magnetic resonance imaging, or diagnostic hysteroscopy; (2) not accompanied with blood system diseases, liver or kidney dysfunction; (3) no contraindications for hysteroscopic surgery. Exclusion criteria included the following: (1) patients with acute systemic or pelvic inflammatory diseases; (2) complicated with malignant tumor of reproductive tract; (3) recent history of uterine perforation (within 3 month). In total, 740 patients met the inclusion criteria, including 131 cases of submucosal myomas, 85 cases of polyps and 524 cases of RPOC. Written information consent was provided by the participants. Table 1 illustrates the demographic characteristics and the procedure indication.

\section{Surgical procedure}

Hysteroscopic resection of the intrauterine lesions was performed by experienced gynecologists. After general anesthesia, the locations of the intrauterine lesions and the uterine cavity were visualized using a diagnostic hysteroscope. Distension of uterine cavity was obtained using $0.9 \%$ normal saline, and the 
intrauterine pressure was automatically controlled by an electronic irrigation and suction device. The pressure was set at 100 $140 \mathrm{mmHg}$. Then, we performed all procedures using the MyoSure hysteroscopic tissue removal system (Hologic Inc., Marlborough, MA). If required, $10 \sim 20 \mathrm{mg}$ furoxime should be used approximately $10 \mathrm{~min}$ after the operation to prevent hyperhydration syndrome in patients with an expected operative time of greater than $30 \mathrm{~min}$, and oxytocin solution injection through the cervical stroma could be used to stop bleeding. Patients with fertility desire were injected with $3 \sim 5 \mathrm{ml}$ selfcrosslinking sodium hyaluronate gel (Changzhou Paget) in the uterine cavity after surgery to prevent intrauterine adhesion. The patient's vital signs, vaginal bleeding, abdominal pain and other conditions should be closely observed. For patients with more postoperative vaginal bleeding ( $\$ 100 \mathrm{ml}$ ) and poor uterine contractile function, intravenous oxytocin infusion can be used to promote contraction and hemostasis. Patients who had vaginal bleeding for more than 7 days before operation were administered

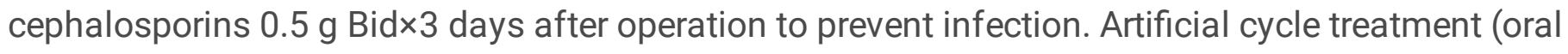
estradiol valerate tablets $2 \sim 3 \mathrm{mg}$ Bid for 21 days followed by progesterone capsule $200 \mathrm{mg}$ Qd for 6 days for a total of $1 \sim 3$ cycles) was administered to patients with fertility requirements to promote intimal growth.

\section{Follow-up}

The patient was ordered to undergo pelvic ultrasonography for auxiliary diagnosis of the intrauterine lesions, and the lesions were cleared by hysteroscopy when necessary. The follow-up contents included the alleviation of abnormal uterine bleeding and menstrual recovery of the patient. Evaluation criteria were as follows: (1) Satisfactory efficacy: the lesion was cleared completely during the operation, postoperative vaginal bleeding stopped within 2 weeks, menstruation returned to normal, and menstrual volume returned to normal or decreased compared with that prior to the operation. (2) Unsatisfactory curative effect: incomplete intraoperative lesion removal that requires reoperation or drug intervention, irregular vaginal bleeding caused by residual lesions after operation, menstrual volume increased to levels greater than that noted before surgery, and evidence of intrauterine adhesions.

\section{Results}

\section{Operative conditions}

During the study period, 740 women with intrauterine lesions were successfully resected using MyoSure. Operative conditions are provided in Table 2. The operative time of 1 patient with RPOC was greater than 60 min. During the operation, typical fine moist rale and blood gas analysis results indicated hyperhydration syndrome. After using furosemide for diuresis, the symptoms alleviated. The remaining patients had stable vital signs during and after the operation, and no complications, such as uterine perforation or infection, occurred.

The CRRs of types $0, \nabla$, and $\nabla$ and mixed type submucosal myomas were $100.0 \%(45 / 45), 96.00 \%(48 / 50)$, $85.67 \%(13 / 15)$ and $75.00 \%(9 / 12)$, respectively. Lesions in 7 patients were not entirely cleared. Among

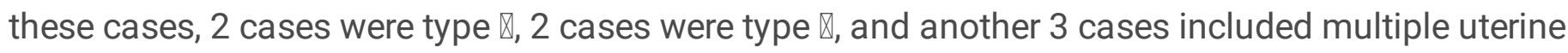


fibroids. To avoid hyperhydration syndrome due to a prolonged operation time, some patients visit again 1 month to 3 months later for a second hysteroscopy and the removal of residual lesions. The CRR of RPOC is $98.28 \%$ (515/524). Specifically, 9 cases were not entirely cleared (6 cases of placenta residue or implantation and 3 cases of incomplete abortion, including 2 cases of lesions located in the incision site of the lower uterine segment). During the operation, the lesions were scattered around the walls of the uterine cavity, and active bleeding and blurred vision were noted. Given the prolonged operative time, we immediately interrupted the procedure to prevent complications, such as uterine perforation or hyperhydration syndrome. All the patients underwent hysteroscopy reexamination, and the residual pregnancy lesions were cleared after 1 month to 3 months.

\section{Clinical outcomes}

Patients with increased menstrual volume, prolonged menstrual period or irregular vaginal bleeding before surgery all returned to normal menstruation. The postoperative symptom relief rate was $98.85 \%$ $(518 / 524)$ in the patients with RPOC. Six patients had unsatisfied clinic outcomes as these patients continued to experience vaginal bleeding for more than 2 weeks after the operation. Among these patients, pelvic ultrasonography reexamination indicated mixed intrauterine echo, which is indicative of residual pregnancy lesions. We performed second hysteroscopy and removed the residual lesions.

Hysteroscopy was performed in 96 patients after surgery (10 after submucosal myomectomy, 85 after surgery for RPOC, and 1 after removal of endometrial polyps). Intrauterine adhesions were found in 37 patients, accounting for $5.0 \%$ (37/740). Among them, 3 patients exhibited mild adhesion (mild AFS score of 2 points) after submucosal myomectomy. The remaining 34 cases had severe adhesion after the surgery for RPOC, among which 14 cases had underwent several rounds of dilatation and curettage in other hospitals (severe AFS score of $9 \sim 10$ points). Another 20 patients had mild adhesion (AFS scores ranging from 4 to 8 points).

\section{Birth outcomes}

A total of 359 patients were effectively followed up after operation (76 cases after submucosal myomectomy, 235 cases after surgery for RPOC, and 48 cases after removal of endometrial polyps). The conception rate was $47.91 \%$ (172/359), including 26 cases after submucosal myomectomy, 123 cases after surgery for RPOC, and 23 cases after removal of endometrial polyps. The birth outcomes are presented in Table 3. The interval between conception and operation ranged from 1 to 34 months with an average of 11.1 months. The live birth rate was $59.30 \%$ (102/172), including 52 from vaginal deliveries and 50 from cesarean deliveries, of whom 5 patients ( 4 after surgery for RPOC and 1 after removal of endometrial polyps) received manual placenta removal during labor or operation, 1 patient underwent postpartum hemorrhage, and 1 patient retained residual placenta.

\section{Discussion}


Benign intrauterine lesions mainly include submucosal myomas, endometrial polyps, and RPOC, which are common causes for abnormal uterine bleeding in women of childbearing age and are also one of the most important reasons leading to infertility. Bipolar electrical resection is often used in these lesions, which adopts active and passive electrotomy to effectively remove the lesions. However, cervical trauma or cervical insufficiency may occur due to mechanical augmentation of the cervical during the operation adapting to the relatively wide endoscopic sheath (outer diameter of 9 to $10 \mathrm{~mm}$ ). The cutting depth of the electrosurgical ring is difficult to control, which increases the risk of uterine perforation and requires high technical skill of the surgeon. Additionally, to ensure the clarity of the operative field, the lesion fragments need to be removed repeatedly during the operation, which prolongs the operation time and increases the risk of infection. In addition, due to the use of electricity, secondary damage to the endometrium of the normal site around the lesion is difficult to avoid, which may result in intrauterine adhesions or other complications that may affect the patients' reproductive outcomes. With the development of hysteroscopic cold knife technology, the efficacy of the power cutting cold knife instrument represented by MyoSure has been gradually recognized. This method offers the advantages of high efficiency and safety in the treatment of benign intrauterine lesions and has gradually become a new treatment choice for women of childbearing age [3,4].

Uterine fibroids are the most common benign pelvic tumors in women of childbearing age with an incidence of approximately $20 \%$ to $25 \%$ [6]. Most asymptomatic patients have submucosal myomas with an incidence of $5.5 \%$ to $16.6 \%$ [8]. MyoSure demonstrates high efficiency and safety in the treatment of submucosal fibroids [7,9]. Emanuel, Lukes, Doggen, Noventa et al. have reported that CRRs of type 0 and type $\otimes$ submucosal myoma range from $63.60 \%$ to $96.23 \%$ [10-14]. Compared to the bipolar electrical resection, MyoSure's operative time for submucosal fibroids was reduced by $61 \%$, and the rate of intraoperative hyperhydration syndrome is lower than that of the resection group $(0.02 \%$ vs $10.10 \%)$. In type $\otimes$ submucosal fibroids, greater than $50 \%$ of the fibroids are located within the muscle layer.

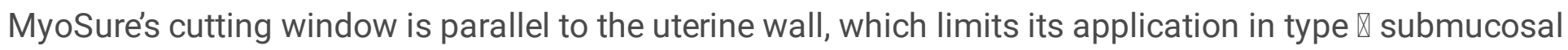
fibroids. However, via contraction of the muscle or through the use of oxytocin, the fibroid part within muscle layer can be made into a cavity for resection. The contractions also have the effect of hemostasis. In our study, CRRs of three types of submucosal myomas ranged from $72.73 \%$ to $100 \%$, and the CRR of type $\nabla$ submucosal myoma was also $92.31 \%$. No intraoperative complications occurred, and all the patients' abnormal uterine bleeding symptoms were alleviated.

Endometrial polyps are one of the most common endometrial lesions in women of childbearing age with an incidence of approximately $24 \%$ to $26 \%$ [15]. The clinical manifestations mostly included intermenstrual bleeding or abnormal uterine bleeding. Some patients sought medical treatment for asymptomatic intrauterine lesions. Suction and curettage or hysteroscopic excision of the lesion are commonly used for polyp's section. However, given that the operation is performed under conditions of blind vision, simple suction and curettage may cause the lesions to be missed or damage the endometrium. Hysteroscopy can be performed under direct sight, but it is difficult to completely remove the pedicle of the lesion, which may lead to frequent recurrence. MyoSure can gradually remove the polyp from the surface to the pedicle with a high-speed rotary blade and can also reduce the risk of endometrial 
damage due to the limitation of the site of action. Previous studies demonstrated that MyoSure's CCR for polyps was $95.2 \%$ to $99.7 \%$ [16]. In our study, 85 cases of endometrial polyps were all completely cleared, and abnormal uterine bleeding symptoms were alleviated in all patients, yielding an efficacy satisfaction rate of $100 \%$. In particulate, 19 patients with intact hymen underwent MyoSure surgery in the condition of preserving the hymen integrity. The outside diameter of MyoSure's sheath is only $6.25 \mathrm{~mm}$, and the polyp fragments can be removed quickly in vitro, which avoid the damage to hymen from repeated actions. Thus, MyoSure exhibits unique advantages in the treatment of these intrauterine lesions in individuals without sexual activity.

RPOC means that all or part of the pregnancy tissue remains in the uterine cavity because it did not discharge in time. This condition primarily occurs secondary to missed abortion, incomplete abortion, induced labor in middle or late pregnancy and other adverse pregnancy outcomes. RPOC mostly manifest as abnormal vaginal bleeding after delivery and can also lead to secondary infection or even affect the subsequent birth outcomes [17]. Hysteroscopy is the most effective treatment, which has the ability to directly visualize the lesion site and lesion size, thus reducing the risk of missing the lesion. MyoSure can attract and remove the residual pregnancy lesions through the side of the cutting window, which can reduce the incidence of uterine perforation for perinatal soft uterus that has not returned to normal shape. In addition, due to the rich intrauterine blood supply during pregnancy and the opening of blood sinuses during the operation, the patient's surgical tolerance was reduced; however, MyoSure reduces the operative time and improves the safety of the operation. In our study, the CCR reached $98.28 \%(515 / 524)$ in 524 patients with RPOC. No uterine perforation occurred during the operation, and only 1 patient developed hyperhydration syndrome. In addition, for lesions in special areas, such as the uterine horn or cesarean section scar diverticulum, MyoSure's endoscopic sheath is narrow, and the operation hole is long and narrow. Thus, the device can easily reach narrow intrauterine areas or deal with refractory lesions. Keriakos applied MyoSure to patients with cornual pregnancy, and all the residual pregnancy tissues were completely removed [18]. In our study, patients with incomplete abortion also had lesions located at the uterine horn, and their CCRs reached $100 \%$. In addition, the abnormal vaginal bleeding symptom was alleviated in all patients. However, the application of MyoSure in cesarean scar pregnancy has been rarely reported. Our study included 35 patients with cesarean scar pregnancy. After the operation, no one exhibited obvious residual pregnancy tissues. Using the pressure suction device, lesions in the sag of uterine cavity can also be attracted and cleared simultaneously.

Recently, more and more women in childbearing age see the doctor for the treatment of intrauterine lesions. For patients with fertility requirements, the protection of fertility function during the procedure is particularly valued. Studies have shown that the incidence of intrauterine adhesions caused by repeated intrauterine operation is as high as $25 \% \sim 30 \%$, while the incidence of pregnancy after separation of adhesions is only 22.5\% 33.3\% [19]. However, MyoSure uses mechanical energy to remove the lesions, which can abtain high complete resection rate. Also, the limitation of the site of action can reduce the damage to the endometrium, therefore can improve the reproductive outcomes. Wei Li et al. performed lesion removal using MyoSure in 60 patients with refractory RPOC. The postoperative menstrual recovery time and endometrial thickness in the MyoSure group were both improved compared with that in the 
electrotomy group. In our study, endometrial repair was evaluated through the appearance of postoperative intrauterine adhesions. The incidence of intrauterine adhesions after MyoSure surgery was $5.0 \%$ (37/740), but most of them were mild to moderate adhesions. In total, 14 patients exhibited severe adhesion. Some of these patients have implanted residual pregnancy tissue, whereas others previously underwent several intrauterine procedures. Thus, intrauterine adhesions or endometrium damage occurred prior our operation, and RPOC might cover the adhesion. Given the low estrogen levels, the endometrium was imperfectly repaired, thereby creating further adhesion. After follow-up for three years, $359(47.91 \%)$ patients got pregnant, among which the natural conception rate was $84.88 \%(146 / 172)$ and the live birth rate reached $59.30 \%$ (102/172). Only 5 patients were complicated with pregnancy complications, which include placenta accreta, postpartum hemorrhage and RPOC, which account for $4.9 \%(5 / 102), 0.98 \%(1 / 102)$ and $0.98 \%(1 / 102)$ respectively in the routine.

In summary, MyoSure has the advantages of safety and efficiency in the treatment of intrauterine lesions. The method protects the endometrium, thereby representing a new choice for the treatment of benign intrauterine lesions in women of childbearing age.

\section{Declarations}

Author contributions: YJ contributed to the conception, draft writing and data analysis. GX, LC, YJ, WY, ZF, $S X, Y Y, Y S$ and $Z X$ contributed to collect and analyze the data. XS contributed to collect data, revise the draft and manuscript. All authors read and approved the final version of the manuscript.

\section{Acknowledgments}

We thank all of our patients for their participation in this study.

Conflict of interest: None.

Funding: 1. The Project of Hunan Provincial Natural Science Foundation Grant No. 2018JJ3782, supported by Hunan province natural science foundation; 2. China Scholarship Fund, CSC No. 201806375049, supported by China scholarship fund.

Synopsis: The MyoSure hysteroscopic tissue removal system is a safe, effective and endometrium protecting treatment for benign intrauterine lesions for women of childbearing age.

\section{References}

1. Donnez J, Dolmans MM, "Uterine fibroid management: from the present to the future." Human reproduction update, 2016, 22(6): 665-686.

2. Klatsky PC, Tran ND, Caughey AB. Fibroids and reproductive outcomes: a systematic literature review from conception to delivery[J]. Am J Obstet Gynecol, 2008, 198(4): 357-366. 
3. Friedman J.A, Wong J.M.K. Hysteroscopic myomectomy: a comparison of techniques and review of current evidence in the management of abnormal uterine bleeding[J]. Current opinion in obstetrics \& gynecology, 2018, 30(4): 243-251.

4. Emanuel MH. New developments in hysteroscopy[J]. Best Pract Res Clin Obstet Gynaecol, 2013, 27(3): 421-429.

5. Cohen, S, Greenberg JA, "Hysteroscopic morcellation for treating intrauterine pathology." Reviews in obstetrics \& gynecology, 2011, 4(2): 73-80.

6. Capmas P, Levaillant JM, et al, "Surgical techniques and outcome in the management of submucous fibroids." Current opinion in obstetrics \& gynecology, 2013, 25(4): 332-8.

7. Camanni M, Bonino L, Delpiano EM, et al. Hysteroscopic management of large symptomatic submucous uterine myomas. J Minim Invasive Gynecol, 2010, 17(1):59 『65》

8. Klatsky PC, Tran ND, Caughey AB. Fibroids and reproductive outcomes: a systematic literature review from conception to delivery[J]. Am J Obstet Gynecol, 2008, 198(4): 357-366.

9. Varasteh NN, Neuwirth RS, Levin B, Keltz MD. Pregnancy rates after hysteroscopic polypectomy and myomectomy in infertile women. Obstet Gynecol, 1999; 94:168-171.

10. Rubino RJ, Lukes AS. Twelve-month outcomes for patients undergoing hysteroscopic morcellation of uterine polyps and myomas in an office or ambulatory surgical center[J]. J Minim Invasive Gynecol, 2015, 22(2): 285-290.

11. Dongen $\mathrm{H}$, Emanuel MH, Wolterbeek R. Hysteroscopic morcellator for removal of intrauterine polyps and myomas: a randomized controlled pilot study among residents in training[J]. Minim Invasive Gynecol, 2008, 15(4): 466-471.

12. Noventa M, Ancona E, Quaranta M. Intrauterine Morcellator Devices: The Icon of Hysteroscopic Future or Merely a Marketing Image? A Systematic Review Regarding Safety, Efficacy, Advantages, and Contraindications[J]. Reprod Sci. 2015, 22(10): 1289-1296.

13. Emanuel MH. New developments in hysteroscopy[J]. Best Pract Res Clin Obstet Gynaecol, 2013, 27(3): 421-429.

14. Liang Y, Ren Y, Wan Z. Clinical evaluation of improved MyoSure hysteroscopic tissue removal system for the resection of type II submucosal myomas[J]. Medicine (Baltimore), 2017, 96(50): e9363.

15. Yasuda $M$, Katoh $T$, Hori $S$, et al. Endometrial intraepithelial carcinoma in association with polyp: review of eight cases[J]. J Diagn Pathol, 2013, 8(21):25-26

16. Georgiou D, Tranoulis A, Jackson TL. Hysteroscopic tissue removal system (MyoSure) for the resection of polyps, sub-mucosal leiomyomas and retained products of conception in an out-patient setting: A single UK institution experience[J]. Eur J Obstet Gynecol Reprod Biol, 2018, 231: 147-151.

17. Golan A, Dishi M, Shalev A. Operative hysteroscopy to remove retained products of conception: novel treatment of an old problem[J]. J Minim Invasive Gynecol, 2011, 18(1): 100-103.

18. Keriakos. MyoSure in management of cornual ectopic pregnancy. [J]. Journal of obstetrics and gynaecology: the journal of the Institute of Obstetrics and Gynaecology, 2016, 36(4): 426-427. 
19. Yu D, Wong YM, et al. "Asherman syndrome-one century later." Fertility and sterility, 2008, 89(4): 75979.

\section{Tables}

Table 1 Demographic characteristics and indication of procedure

\begin{tabular}{|ll|}
\hline Variables & Entire cohort \\
\hline Patient age (years) & \\
\hline Submucosal leiomyomas & $35.4(21-53)$ \\
\hline Polyps & $29(17-48)$ \\
\hline RPOC & $31.5(18-38)$ \\
\hline Reason for treatment & \\
\hline Abnormal uterine bleeding & \\
\hline Submucosal leiomyomas & $86(65.65 \%)$ \\
\hline Polyps & $49(57.65 \%)$ \\
\hline RPoC & $356(67.94 \%)$ \\
\hline Infertility & \\
\hline Submucosal leiomyomas & $44(33.59 \%)$ \\
\hline Polyps & $36(42.35 \%)$ \\
\hline Postmenopausal & \\
\hline Submucosal leiomyomas & $1(0.76 \%)$ \\
\hline
\end{tabular}

Table 2 Operative conditions 


\begin{tabular}{|c|c|}
\hline Lesion characteristics & Entire cohort \\
\hline \multicolumn{2}{|l|}{ Maximal diameter (cm) } \\
\hline Submucosal leiomyomas & $2.75 \pm 1.11$ \\
\hline Polyps & $1.52 \pm 0.80$ \\
\hline RPOC & $3.10 \pm 1.24$ \\
\hline \multicolumn{2}{|l|}{ Leiomyomas type } \\
\hline Type 0 & 45 (36.89\%》 \\
\hline Type $\nabla$ & $50(40.98 \% \rrbracket$ \\
\hline Type $\otimes$ & $15(12.30 \% \rrbracket$ \\
\hline Types $0, \nabla$ and $\nabla$ & $12(9.84 \% \square$ \\
\hline \multicolumn{2}{|l|}{ Polyps type } \\
\hline Single & $49(57.65 \%)$ \\
\hline Multiple & $36(42.35 \%)$ \\
\hline \multicolumn{2}{|l|}{ Blood loss (ml) } \\
\hline Submucosal leiomyomas & $17.09 \pm 14.87$ \\
\hline Polyps & $11.78 \pm 10.89$ \\
\hline RPOC & $29.50 \pm 13.74$ \\
\hline \multicolumn{2}{|l|}{ Operative time (min) } \\
\hline Submucosal leiomyomas & $42.37 \pm 19.33$ \\
\hline Polyps & $20.96 \pm 8.99$ \\
\hline RPOC & $30.47 \pm 14.04$ \\
\hline \multicolumn{2}{|c|}{ Complete resection rate (CRR) } \\
\hline \multicolumn{2}{|l|}{ Submucosal leiomyomas } \\
\hline Type 0 & $100.0 \%(45 / 45)$ \\
\hline Type $\nabla$ & $96.00 \%(48 / 50)$ \\
\hline Type $\nabla$ & $85.67 \%(13 / 15)$ \\
\hline Types $0, \nabla$ and $\otimes$ & $75.00 \%(9 / 12)$ \\
\hline Polyps & $100.0 \%(85 / 85)$ \\
\hline RPOC & $98.28 \%(515 / 524)$ \\
\hline
\end{tabular}


Table 3 Birth outcomes

\begin{tabular}{|ll|}
\hline Birth outcomes & Entire cohort \\
\hline Conception & \\
\hline Submucosal leiomyomas & $21(14.38 \%)$ \\
\hline Polyps & $14(9.59 \%)$ \\
\hline RPOC & $111(76.03 \%)$ \\
\hline Assisted reproduction & \\
\hline Submucosal leiomyomas & $5(19.23 \%)$ \\
\hline Polyps & $9(34.62 \%)$ \\
\hline RPOC & $12(46.15)$ \\
\hline Live birth & \\
\hline Vaginal delivery & $52(50.98 \%)$ \\
\hline Cesarean delivery & $50(49.02 \%)$ \\
\hline Preterm delivery & \\
\hline Vaginal delivery & $20(90.91 \%)$ \\
\hline Cesarean delivery & $2(9.09 \%)$ \\
\hline Induced labor & $7(4.07 \%)$ \\
\hline Missed abortion & $4(2.33 \%)$ \\
\hline Abortion & $30(17.44 \%)$ \\
\hline Cesarean scar pregnancy & $1(0.58 \%)$ \\
\hline Ectopic pregnancy & $1(0.58 \%)$ \\
\hline Biochemical pregnancy & $1(0.58 \%)$ \\
\hline Pregnant & $4(2.33 \%)$ \\
\hline
\end{tabular}

